\title{
Chemical abundances of Planetary Nebulae in M 33^
}

\author{
L. Magrini ${ }^{1}$, M. Perinotto ${ }^{1}$, A. Mampaso ${ }^{2}$, and R. L. M. Corradi ${ }^{3}$ \\ 1 Dipartimento di Astronomia e Scienza dello Spazio, Universitá di Firenze, L.go E. Fermi 2, 50125 Firenze, Italy \\ e-mail: laura@arcetri.astro.it \\ 2 Instituto de Astrofísica de Canarias, c. vía Láctea s/n, 38200, La Laguna, Tenerife, Canarias, Spain \\ ${ }^{3}$ Isaac Newton Group of Telescopes, Apartado de Correos 321, 38700 Santa Cruz de La Palma, Canarias, Spain
}

Received 16 April 2004 / Accepted 27 June 2004

\begin{abstract}
Using spectroscopic data presented in Magrini et al. (2003), we have analyzed with the photoionization code CLOUDY 94.00 (Ferland et al. 1998) 11 Planetary Nebulae belonging to the spiral galaxy M 33. Central star temperatures and nebular parameters have been determined. In particular the chemical abundances of $\mathrm{He} / \mathrm{H}, \mathrm{O} / \mathrm{H}, \mathrm{N} / \mathrm{H}, \mathrm{Ar} / \mathrm{H}$, and $\mathrm{S} / \mathrm{H}$ have been measured and compared with values obtained via the Ionization Correction Factors (ICF) method, when available. Chemical abundance relationships have been investigated; in particular, a correlation between N/H and N/O similar to the Galactic one (Henry 1990), and a feeble anti-correlation between $\mathrm{O} / \mathrm{H}$ and N/O have been found. A gradient in O/H across the disc of M 33 is consistent with the one found from $\mathrm{H}$ II regions in this galaxy (Vílchez et al. 1988). Further studies in the outer parts of M 33 are however needed to ascertain this point. The present result shows that oxygen and helium abundances (with lower accuracy also nitrogen, argon and sulphur) can be actually estimated from the brightest PNe of a galaxy, even if the electron temperature cannot be measured. We also found that the oxygen abundance is quite independent of the absolute magnitude of the PN and consequently the brightest $\mathrm{PNe}$ are representative of the whole PN population. This represents an important tool for measuring the metallicity of galaxies at the time of the formation of PNe progenitors.
\end{abstract}

Key words. ISM: planetary nebulae: individual: M 33 - galaxies: individual: M 33 - galaxies: abundances - Local Group

\section{Introduction}

Chemical abundances in extragalactic Planetary Nebulae (PNe) have been derived so far only in a small number of galaxies. Inside the Local Group they are: the irregular galaxies LMC, SMC (cf. Leisy et al. 2000; Jacoby \& De Marco 2002), and NGC 6822 (Richer \& McCall 1995), the spheroidal and dwarf spheroidal galaxies Fornax (Maran et al. 1984), Sagittarius (cf. Walsh et al. 1997), NGC 185, and NGC 205 (Richer \& McCall 1995), the elliptical galaxy M 32 (Stasinska et al. 1998) and finally the two spiral galaxies M 31 and M 33 which have been studied by Jacoby \& Ciardullo (1999, hereafter JC 99) and Magrini et al. (2003, hereafter M03), respectively. There is a substantial lack of information about chemical abundances of PNe in dwarf irregular galaxies of the Local Group (e.g. Sextans B, Sextans A, Leo A, IC 10), where no PN has been investigated so far. Outside the LG, only PNe in the giant elliptical galaxy NGC 5128 (Centaurus A) at the distance of $3.5 \mathrm{Mpc}$ were analyzed by Walsh et al. (1999).

In most of the previous cases, with the exception of NGC 5128, the abundances have been obtained by direct

^ Based on observations obtained at the $4.2 \mathrm{~m}$ WHT telescope operated on the island of La Palma by the Isaac Newton Group in the Spanish Observatorio del Roque de Los Muchachos of the Instituto de Astrofisica de Canarias. determination of the $T_{\mathrm{e}}$, derived from the [O III] $\lambda \lambda$ 4363/ $5007 \AA$ or, in the worst cases when the $\lambda 4363 \AA$ is not measured, using its upper limit, thus obtaining lower limits for the [O III] ion abundance and from this the total $\mathrm{O}$ abundance, which was calculated from the ICF (Ionization Correction Factors) procedure (cf. e.g. Kingsburgh \& Barlow 1994, hereafter KB 94).

In M 31, in addition to using the ICF method, JC 99 modeled the observed $\mathrm{PNe}$ using the photoionization code CLOUDY 90 (Ferland et al. 1998) with simplified assumptions: blackbody central stars and spherical nebulae with constant density. They derived abundances and central star parameters for $15 \mathrm{PNe}$ of $\mathrm{M} 31$. In eight cases they could compare the results from the nebular models built with CLOUDY with abundances based on the ICF method. They found good agreement between abundances derived with these two different methods. From this comparison, CLOUDY proved to be a useful tool for measuring chemical abundances, particularly when direct electron temperature measurements are not available. M 03 studied 48 emission line objects in M 33 spectroscopically, and recognized, via diagnostic diagrams, 26 of them to be bona fide PNe. They presented the observed fluxes for 42 of the 48 objects, deriving chemical abundances with the ICF method for the three brightest PNe.

In the present paper, we re-analyze 11 of the $26 \mathrm{PNe}$ presented by M03 using the available spectroscopic data and 


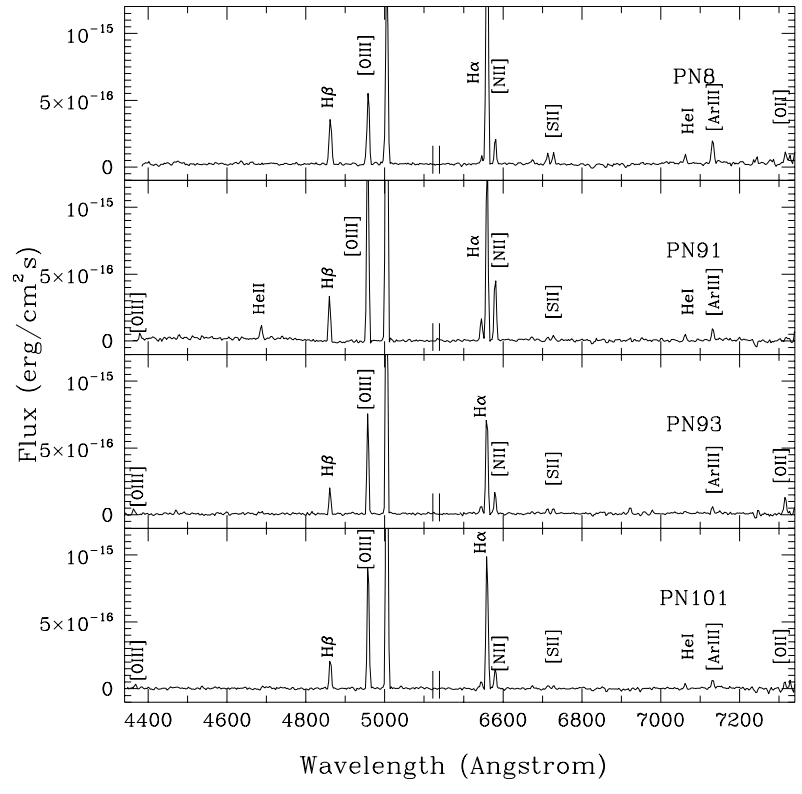

Fig. 1. Characteristic spectra of PNe in M 33 obtained by M 03 with an exposure time of $8.7 \mathrm{~h}$ with the WHT.

CLOUDY 94.00 (Ferland et al. 1998). The three PNe already studied with ICF by M 03 are also included, in an effort to clarify their observed low N/O.

In Sect. 2 we summarize the observations and data reduction. In Sect. 3 the derivation of nebular and stellar properties is illustrated. In Sect. 4 we discuss the derived quantities and correlations among them. The summary and conclusions are in Sect. 5.

\section{Observations and data reduction}

The observations were obtained at the $4.2 \mathrm{~m}$ WHT telescope (La Palma, Spain) in October, 2001 using AF2/WYFFOS, with a setting producing a dispersion of $3.0 \AA$ per pixel in the spectral range $4300 \AA$ to $7380 \AA$. A detailed description of the spectroscopic data and of the reduction procedure are in M 03 .

\section{The modelling procedure}

By using a photoionization model such as CLOUDY it is possible to overcome the lack of direct information on the electron temperature in a PN due to the faintness of the relevant forbidden lines: the [O III] $\lambda 4363$ and the [ $\mathrm{N}$ II] $\lambda 5755$ emission lines, which are hard to detect in extragalactic PNe. On the other hand, the use of CLOUDY requires as input the energy distribution of the ionizing radiation of the central star, its luminosity and the nebular geometry. The distance to the object must thus be known. In our case the distance is available if we adopt for all PNe in M 33 the distance of the galaxy, equal to $840 \pm$ $90 \mathrm{kpc}$ (Magrini et al. 2000, hereafter M 00). As concerning the energy distribution of the central stars the usual simplification is to assume them to behave as blackbodies (BB), while for a better accuracy stellar atmosphere models are needed (cf. Morisset 2004). In this respect, an illustrative test was carried out by Howard et al. (1997). They tested the effects on derived abundances and central star temperatures of a variety of model atmospheres and blackbodies in a set of Galactic halo PNe. They found that nebular line strengths are relatively insensitive to atmospheric model details and that blackbody spectra are by and large suitable for representing continuum of the central star. On the other hand Armsdorfer et al. (2002) checked the effects of modelling a PN central star with a blackbody instead of with a model atmosphere in the particular case of the spatially resolved round Galactic PN NGC 2438. They found that the $\mathrm{BB}$ assumption leads to underestimating the [O III] $\lambda 5007$ line and to overestimating the He II $\lambda 4686$ line strength. Therefore the assumption of BB central stars might introduce uncertainties in the determination of chemical abundances. In the next sections, we will also check in a specific case the BB assumption vs. the use of a model atmosphere. We will see that the differences in the resulting abundances are quite small.

The aim of our procedure is to match the intensities of the observed emission lines with those predicted by CLOUDY. The spirit of our procedure is similar to the one adopted by JC 99 to determine the chemical abundances of PNe in M 31. In the following we briefly describe how our analysis works.

CLOUDY needs the following input parameters: the energy distribution and luminosity of the central star, the nebular geometry, the electron density, and the chemical abundances. Because of the lack of information for our objects on the morphology and on the density distribution, we set the geometry to be spherical and the density to a constant value, although we will explore the effect of a $r^{-2}$ density distribution in some PNe. The energy distribution of the central star was set to that of a BB. For the three PNe (PN 91, 93, 101) with the best $\mathrm{S} / \mathrm{N}$ spectra and with chemical abundances derived also with the ICF method (M03), we will compare nebular abundances from the blackbody central star with those from the model atmospheres by Rauch (2003, see Table 2).

The first run was done with the input parameters set as follows. The BB temperature was derived using Ambartsumian's method (Ambartsumian 1932), i.e. using the $\mathrm{He} \mathrm{II}(4686) / \mathrm{H} \mathrm{I}(4861)$ line flux ratio, or the $([\mathrm{O}$ III $](4959)+[\mathrm{O}$ III] $(5007)) / \mathrm{He}$ II(4686)) line ratio method (Gurzadyan 1988) for medium and high excitation objects, respectively. Medium and high excitation is recognized from the observed line ratio $\mathrm{He}$ II(4686)/H I(4861). Following Gurzadyan (1996) we call high excitation PNe those with He II(4686)/H I $(4861)>0.15^{1}$.

The luminosity of the central star was set to reproduce the observed absolute flux of the [O III] $\lambda 5007$ nebular line, measured by Magrini et al. (2000) via aperture photometry, and reddening-corrected with $E(B-V)=0.07$ (van den Bergh 2000). We made an effort to correct each $\lambda 5007 \AA$ [O III] flux with the individual reddening coming from the Balmer decrement. We saw that in some cases the corrected luminosity was

\footnotetext{
${ }^{1}$ Gurzadyan (1988) distinguishes between low and high excitation objects. We prefer to use the term "medium" instead of low because when the He II $\lambda 4686$ line is measured the excitation cannot be really be regarded as low. We think the specification "low" is more appropriate for PNe where the line is not detectable.
} 
Table 1. Central star temperatures and nebular parameters. Chemical abundances are expressed in $12+\log (X / \mathrm{H})$. The line fluxes $F_{\mathrm{H} \alpha}$ and $F_{[\mathrm{OIII}]}$ as observed by M00. The adopted reddening correction is $E(B-V)=0.07$ (van den Bergh 2000).

\begin{tabular}{|c|c|c|c|c|c|c|c|c|c|c|c|c|c|}
\hline $\begin{array}{r}\text { Id } \\
\text { M } 00\end{array}$ & $\begin{array}{r}d \\
\operatorname{arcmin}\end{array}$ & $\begin{array}{l}F_{\mathrm{H} \alpha} \\
10^{-15}\end{array}$ & $\begin{array}{l}F_{[\mathrm{OIII}]} \\
/ \mathrm{cm}^{2} \mathrm{~s}\end{array}$ & $\begin{array}{l}T_{\star} \\
\mathrm{K} / 1000\end{array}$ & $\log \left(L_{\star} / L_{\odot}\right)$ & $\begin{array}{l}\log (r) \\
\mathrm{cm}\end{array}$ & $\begin{array}{c}\log \left(N_{\mathrm{e}}\right) \\
\mathrm{cm}^{-3}\end{array}$ & $\mathrm{He} / \mathrm{H}$ & $\mathrm{N} / \mathrm{H}$ & $\mathrm{O} / \mathrm{H}$ & $\mathrm{S} / \mathrm{H}$ & $\mathrm{Ar} / \mathrm{H}$ & $\mathrm{N} / \mathrm{O}$ \\
\hline 8 & 22 & 11.4 & 10.1 & $74 \mathrm{~b}$ & 3.8 & $16<r<18 d$ & 3.1 & 11.05 & 7.07 & 7.94 & 6.38 & 6.11 & -0.87 \\
\hline 18 & 13 & 3.0 & 14.2 & $100 \mathrm{~b}$ & 4.1 & $16<r<17.9 \mathrm{~d}$ & 2.5 & 11.09 & 7.48 & 8.20 & 6.83 & 6.51 & -0.72 \\
\hline 28 & 12 & 2.9 & 13.9 & $142 \mathrm{a}$ & 3.7 & $16<r<17.7 \mathrm{~d}$ & 2.1 & 10.96 & 6.98 & 8.69 & 6.47 & 6.3 & -1.71 \\
\hline 60 & 13 & 1.4 & 3.4 & $160 \mathrm{a}$ & 3.1 & $17 \mathrm{c}$ & 2.9 & 11.04 & 7.67 & 8.69 & 6.57 & 6.31 & -1.02 \\
\hline 65 & 8 & 10.1 & 5.6 & $128 \mathrm{~b}$ & 3.2 & $16<r<17.7 \mathrm{~d}$ & 3.2 & 11.16 & 7.61 & 8.67 & 6.84 & 6.63 & -1.06 \\
\hline 75 & 11 & 4.4 & 12.5 & $96 \mathrm{~b}$ & 3.5 & $16<r<17 \mathrm{~d}$ & 3.9 & 11.14 & 8.03 & 8.49 & 6.81 & 6.41 & -0.46 \\
\hline 91 & 18 & 4.9 & 16.3 & $110 \mathrm{a}$ & 3.6 & $17.0 \mathrm{c}$ & 3.6 & 11.31 & 8.07 & 8.48 & 6.60 & 6.18 & -0.41 \\
\hline 93 & 16 & 3.1 & 7.4 & $96 \mathrm{a}$ & 3.3 & $17.2 \mathrm{c}$ & 3.7 & 10.93 & 7.75 & 8.47 & 6.66 & 6.09 & -0.72 \\
\hline 96 & 9 & 3.1 & 11.9 & $105 \mathrm{~b}$ & 3.5 & $16<r<17.9 \mathrm{~d}$ & 1.9 & 11.06 & 7.43 & 8.76 & 6.44 & 6.33 & -1.33 \\
\hline 101 & 14 & 4.1 & 11.4 & $97 \mathrm{~b}$ & 3.4 & $17 \mathrm{c}$ & 2.5 & 11.07 & 7.33 & 8.72 & 6.18 & 6.27 & -1.56 \\
\hline 125 & 9 & 7.5 & 8.2 & $86 \mathrm{~b}$ & 3.6 & $16<r<17.9 \mathrm{~d}$ & 2.2 & 10.93 & 7.53 & 8.31 & 6.66 & 6.14 & -0.78 \\
\hline \multicolumn{8}{|c|}{ M 33 PNe all (1) } & 11.08 & 7.65 & 8.54 & 6.62 & 6.33 & -0.89 \\
\hline \multicolumn{8}{|c|}{ M 33 PNe with $T_{\star}$ measured (1) } & 11.09 & 7.78 & 8.61 & 6.60 & 6.28 & -0.83 \\
\hline \multicolumn{8}{|c|}{ M 33 H II regions (2) } & 10.92 & 7.34 & 8.55 & 6.95 & - & -1.21 \\
\hline \multicolumn{8}{|c|}{ Galactic PNe non-type I (3) } & 11.05 & 8.14 & 8.69 & 6.91 & 6.38 & -0.55 \\
\hline \multicolumn{8}{|c|}{ Galactic PNe type I (3) } & 11.12 & 8.72 & 8.65 & 6.91 & 6.42 & +0.07 \\
\hline \multicolumn{8}{|c|}{ Galactic H II regions (4) } & 11.00 & 7.57 & 8.70 & 7.06 & 6.42 & -1.13 \\
\hline \multicolumn{8}{|c|}{ LMC PNe non-type I (5) } & 10.96 & 7.46 & 8.35 & 6.81 & 5.95 & -0.90 \\
\hline \multicolumn{8}{|c|}{ LMC PNe type I (5) } & 10.95 & 8.28 & 8.24 & 7.11 & 6.12 & +0.02 \\
\hline \multicolumn{8}{|c|}{ LMC H II regions (6) } & 10.97 & 6.97 & 8.38 & 6.67 & 6.92 & -1.41 \\
\hline
\end{tabular}

\author{
(1) Present paper (average, by number, not by log). \\ (2) Vílchez et al. (1988). \\ (3) KB 94. \\ (4) Dufour (1984). \\ (5) Leisy \& Dennefeld (2004). \\ (6) Dennefeld (1989).
}

substantially above the cutoff of the [O III] luminosity function. Thus we conclude that the de-reddening values from the Balmer decrement suffer from calibration issues. In these cases, while the line fluxes can be considered to be correct, $c \beta$ is should not be used for correcting the photometric fluxes. We have preferred correcting the photometric fluxes using the average extinction towards M 33 .

We varied the external radius from $10^{16} \mathrm{~cm}(0.0032 \mathrm{pc})$ to $10^{18} \mathrm{~cm}(0.32 \mathrm{pc})$. This range corresponds to a reasonable size interval for Galactic PNe. The electron density is evaluated from the [S II] $\lambda \lambda 6717 / 6731$ line ratio. We assumed the average non-type I Galactic PNe abundances from KB 94 as initial chemical abundances and dust grains, with composition and size considered to be typical of Galactic PNe dust (cf. Ferland 1998). The addition of dust to the model does not vary substantially the derived chemical abundances.

In further iterations the following adjustments were made. First the BB $T_{\star}$ was altered to match the $\mathrm{He} \mathrm{I} / \mathrm{He}$ II line ratio. When the He II $\lambda 4686$ line was too weak to be measured, we determined its upper limit so as to derive an upper limit to the temperature of the central star. These cases correspond to $T_{\star}$ marked with $\mathrm{b}$ in Table 1 , while directly measured stellar temperatures are marked with a. In the few cases where the [O II] $\lambda 7325$ doublet was measured the nebular radius was varied to match the observed ratio of the nebular lines [O III] $\lambda 5007$ and [O II] $\lambda 7325$ (cases marked with $\mathrm{c}$ in Table 1, cf. Che \& Köppen 1983) In the other cases, models with different nebular radii were built. For these PNe we found the smallest and the largest radius for which the model converged. Final quantities are the averages of quantities from models spanning these nebular radius intervals (cases marked with $d$ in Table 1).

Subsequently chemical abundances were varied to match the observed and predicted intensities within $5 \%$ for the lines brighter than $\mathrm{H} \beta$ and $20 \%$ for the other lines. The $T_{\mathrm{e}}$ derived from the model is compared with the observed one, when available, i.e. in the three PNe where the [O III] $4363 \AA$ emissionline was measurable. In these $\mathrm{PNe}$, the abundances of $\mathrm{C}$ and $\mathrm{O}$ (the most important coolants) were slightly modified to match the observed $T_{\mathrm{e}}$. The optical depth of the nebula is derived from the hydrogen ionization structure predicted by the 
Table 2. Comparison between CLOUDY abundance determinations with either blackbody central star and a constant density, with model atmosphere by Rauch (2003), and with BB and a density falling off as $r^{-2}$ (two PNe). The last raw gives the ICF abundance determinations (M 03).

\begin{tabular}{clccccc}
\hline \hline $\mathrm{Id}$ & Method & $\mathrm{He} / \mathrm{H}$ & $\mathrm{N} / \mathrm{H}$ & $\mathrm{O} / \mathrm{H}$ & $\mathrm{S} / \mathrm{H}$ & $\mathrm{Ar} / \mathrm{H}$ \\
\hline 91 & $\mathrm{CL}_{\mathrm{BB}}$ & 11.31 & 7.86 & 8.61 & 6.42 & 6.26 \\
& $\mathrm{CL}_{\mathrm{rauch}}$ & 11.20 & 8.01 & 8.64 & 6.52 & 6.25 \\
& $\mathrm{CL}_{\mathrm{BB}+\mathrm{d} \propto r^{-2}}$ & 11.31 & 8.07 & 8.48 & 6.60 & 6.18 \\
& $\mathrm{ICF}_{\mathrm{M} 03}$ & 11.28 & 7.83 & 8.70 & $6.61 \dagger$ & 6.32 \\
\hline 93 & $\mathrm{CL}_{\mathrm{BB}}$ & 11.00 & 7.53 & 8.69 & 6.46 & 6.17 \\
& $\mathrm{CL}_{\mathrm{rauch}}$ & 10.92 & 7.55 & 8.60 & 6.44 & 6.13 \\
& $\mathrm{CL}_{\mathrm{BB}+\mathrm{d} \propto r^{-2}}$ & 10.93 & 7.75 & 8.47 & 6.66 & 6.09 \\
& $\mathrm{ICF}_{\mathrm{M} 03}$ & 11.00 & 7.50 & 8.85 & $6.44 \dagger$ & 6.36 \\
\hline \multirow{2}{*}{101} & $\mathrm{CL}_{\mathrm{BB}}$ & 11.07 & 7.33 & 8.76 & 6.18 & 6.27 \\
& $\mathrm{CL}_{\mathrm{rauch}}$ & 11.14 & 7.34 & 8.70 & 6.13 & 6.22 \\
& $\mathrm{ICF}_{\mathrm{M} 03}$ & 11.15 & 7.21 & 8.72 & $6.32 \dagger$ & 6.45 \\
\hline
\end{tabular}

$\dagger$ See text.

CLOUDY model. The PNe are optically thick, while a few with lower density (PN 28, PN 96, PN 125) were found to be optically thin.

Generally, we need from 10 to 30 iterations to reach convergence, i.e. to reproduce the observed line spectrum within 5-20\%. Well determined quantities are the luminosity of the central star, because distance and absolute [O III] $5007 \AA$ flux are both well known, and the temperature of the central star (when both He I and He II lines are measured). The determination of the nebular radius is instead less reliable, because the measurement of $\lambda 7325 \AA$ [O II] doublet is affected by large observational errors. In two PNe where the [O II] $\lambda 7325$ doublet was measured (PN 91, 93) we introduced the dependence on $r^{-2}$ in the density to obtain better agreement between the observed and predicted $[\mathrm{O} \mathrm{III}] /[\mathrm{O}$ II $]$ ratios. In fact the ratio $[\mathrm{O} \mathrm{III}] /[\mathrm{O}$ II $]$ is not only sensitive to the size of the nebula, but it also depends also on several other parameters, mainly on the density distribution (cf. Che \& Köppen 1983). When $T_{\star}$ is derived from the upper limit of the He II $\lambda 4686$ line, only an upper limit to the helium and other metal abundances can be derived.

The derived $T_{\star}$ and nebular quantities are shown in Table 1. We recall that the $T_{\star}$ derived from the upper limit of the He II $\lambda 4686$ are marked with $b$, whereas the directly measured $T_{\star}$ are marked with a. Derived nebular radii are marked with c, and upper limit to nebular radii with $\mathrm{d}$.

In Table 2 chemical abundances for three PNe computed using ICF (M 03) are compared with current CLOUDY results. Note that for $\mathrm{Ar} / \mathrm{H}$ the ionization correction factors are larger that one because only the [Ar III] $\lambda 7135$ line is detected.

\subsection{Tests of the procedure}

We have tested the accuracy of the method by applying it to seven bright Galactic PNe (M 1-8, M3-1, M 3-5, NGC 2867,

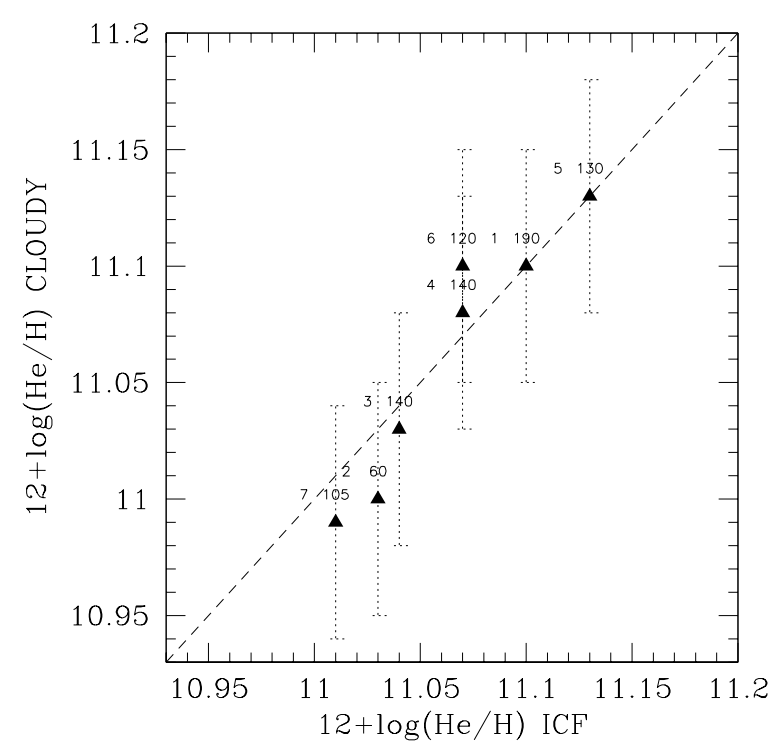

Fig. 2. Comparison of the $\mathrm{He} / \mathrm{H}$ abundance of a sample of Galactic PNe derived with ICF method by KB 94 with $\mathrm{He} / \mathrm{H}$ computed using CLOUDY. Triangles represent $\mathrm{He} / \mathrm{H}$ based on the values of $T_{\star}$ derived in the present work. The id numbers refer to the Galactic PNe: 1- M 1-8, 2- M3-1, 3- M3-5, 4- NGC 2867, $5-\mathrm{NGC} 3195,6-\mathrm{NGC} 7009,7-\mathrm{NGC} 5852$. The derived $T_{\star} / 1000$ is reported near to the corresponding measured $\mathrm{He} / \mathrm{H}$.

NGC 3195, NGC 7009, NGC 5852), using only the emission lines present in our extragalactic PN spectra, and comparing our results with the chemical abundances obtained with the ICF method by KB 94. PNe with central star BB temperatures spanning a large range (from approximately 60000 to $190000 \mathrm{~K}$ ) have been analyzed. For these tests we have used the basic approximations of our model: BB central star and constant density. The results are shown in Figs. 2 and 3. We found a good agreement in the helium abundance, with a rms difference of 0.015 dex (triangles in Fig. 2). The typical rms difference of the two determinations, with CLOUDY and with ICF, of $\mathrm{O} / \mathrm{H}$ is $0.15 \mathrm{dex}$, of $\mathrm{Ar} / \mathrm{H}$ it is approximately 0.1 dex and of $\mathrm{N} / \mathrm{H}$ it is $\sim 0.3 \mathrm{dex}$. In some cases these quantities are underestimated by our method whereas sometimes they are overestimated. On the other hand S/H and N/H appear to be generally underestimated by CLOUDY, with an rms difference of 0.2 dex. A possible explanation for the systematic differences in the determination of $\mathrm{S} / \mathrm{H}$ and $\mathrm{N} / \mathrm{H}$ determination might be related to the fact that for these two elements only low ionization lines are observed. The considered PNe are medium and high excitation objects where these ions represent only a minor fraction of the total elemental abundance. Moreover the ionization potential of $[\mathrm{S} \mathrm{II}]$ is lower than that of $[\mathrm{N} \mathrm{II}]$, which might explain the larger discrepancy in $\mathrm{S} / \mathrm{H}$.

\section{Chemical abundances}

We have applied the procedure described above to $11 \mathrm{PNe}$ of M 33. In four cases we could determine the temperature of the central star within $5000 \mathrm{~K}$ using $\mathrm{He}$ I and $\mathrm{He}$ II lines. In the remaining cases we estimated an upper limit to $T_{\star}$ using upper limits to the He II $\lambda 4686$ flux. Considering the errors in the 

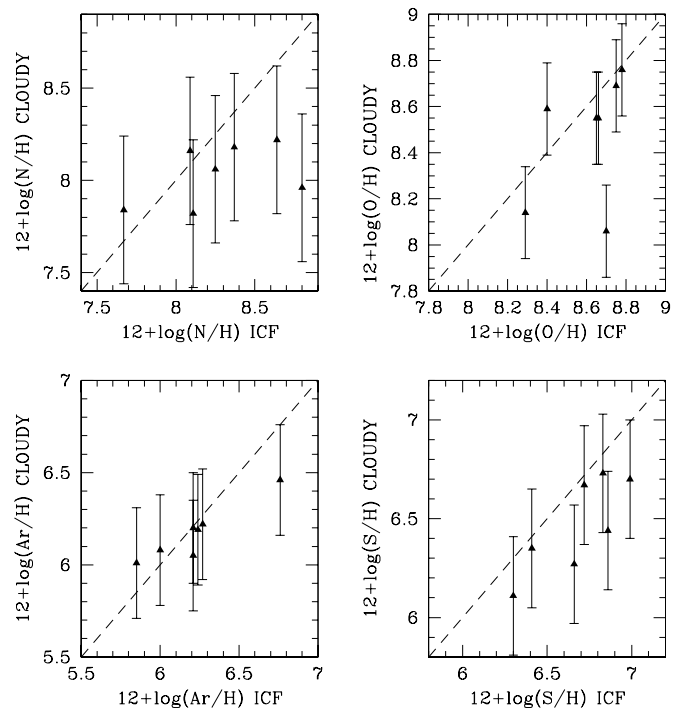

Fig. 3. Comparison of $\mathrm{O} / \mathrm{H}, \mathrm{N} / \mathrm{H}, \mathrm{S} / \mathrm{H}$, and $\mathrm{Ar} / \mathrm{H}$ abundances of seven Galactic PNe derived with the ICF method by KB 94 with the same quantities computed using CLOUDY.

observed fluxes, the uncertainties in the model representation, and the quality in the fit obtained, we estimate typical errors are: $0.05 \mathrm{dex}$ in $\mathrm{He} / \mathrm{H}\left(0.10\right.$ dex when $T_{\star}$ is an upper limit), $0.2(0.3)$ dex in $\mathrm{O} / \mathrm{H}, 0.3(0.4)$ dex in $\mathrm{Ar} / \mathrm{H}$ and $\mathrm{S} / \mathrm{H}$, and $0.4(0.5)$ dex in $\mathrm{N} / \mathrm{H}$. We note that $\mathrm{S} / \mathrm{H}$ is generally underestimated by the CLOUDY method in our test with Galactic PNe, and in addition the highest values of $\mathrm{N} / \mathrm{H}$ appear to be systematically low compared to the values derived using the ICF method.

Chemical abundances, central star parameters, nebular radius and electron density are shown in Table 1. Chemical abundances previously obtained for the three PNe with the ICF method (M 03) are shown in Table 2 together with the new determinations, both for the BB and the model atmosphere (Rauch 2003). The four sets of determinations are consistent within the errors. Since the difference of the chemical abundances derived with CLOUDY using the two stellar ionizing sources are rather small, we used for the remaining PNe a BB central star. The use of a density that depends on $r^{-2}$ with a BB central star produces better agreement between the predicted and observed [O III $] /[\mathrm{O}$ II $]$ ratios. With this model we obtain a higher $\mathrm{N} / \mathrm{H}$ and a lower $\mathrm{O} / \mathrm{H}$, and consequently a higher $\mathrm{N} / \mathrm{O}$ than with ICF method.

$\mathrm{S} / \mathrm{H}$ has been re-derived because of a numerical mistake in the previous work (M03). The difference between the $\mathrm{S} / \mathrm{H}$ values computed with ICF and with CLOUDY (both BB and model atmosphere) is $\sim-0.2$ dex, confirming the result mentioned above that CLOUDY tends to slightly underestimate $\mathrm{S} / \mathrm{H}$.

The comparison in the brightest PNe of M 33 of the abundances derived with the ICF and those from photoionization models confirms the accuracy of the CLOUDY procedure in measuring helium, oxygen, nitrogen and argon abundances.

Stellar evolution theory predicts that $\mathrm{He}$ and $N$ are enhanced in $\mathrm{PNe}$ by nucleosynthesis process, whereas elements heavier than $N$, like $\mathrm{O}$, Ar, $\mathrm{S}$ do not vary significantly from the moment of the formation of their central stars

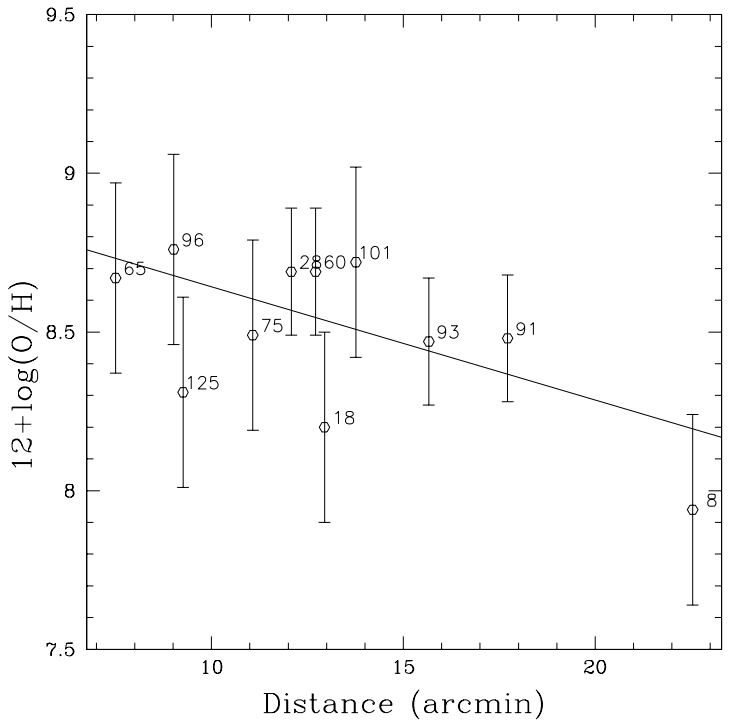

Fig. 4. The galactocentric trend of oxygen in M 33 from 11 PNe, identified by their sequence numbers in Table 1 . The solid line is the galactocentric gradient found from $\mathrm{H}$ in regions by Vílchez et al. (1988) in the same galaxy.

(cf. Iben \& Renzini 1983). In M 33, a comparison of our determinations of the chemical abundances of PNe (particularly for PNe whose $T_{\star}$ could be measured, see Table 1) with those of $\mathrm{H}$ II regions (Vílchez et al. 1988), especially for the best determined elements $\mathrm{He} / \mathrm{H}$ and $\mathrm{O} / \mathrm{H}$ (Table 1), confirms the reliability of the determinations. In fact, as expected, $\mathrm{He} / \mathrm{H}$ (and also $\mathrm{N} / \mathrm{H}$, but remember the larger errors that affect this determination) is enhanced compared to $\mathrm{H}$ II regions while $\mathrm{O} / \mathrm{H}$ is almost unchanged. This indicates that $\mathrm{He}$ and $N$ have undergone nucleosynthesis enrichment in PNe progenitors, as theory predicts. Consequently $\mathrm{N} / \mathrm{O}$ appears to be increased in the M 33 PNe compared with $\mathrm{H}$ II regions.

In Fig. 4 we present the derived oxygen abundances as a function of the galactocentric projected distance. We find a possible oxygen gradient across the disc of M 33, like that detected by Vílchez et al. (1988) for $\mathrm{H}$ II regions. A weighted

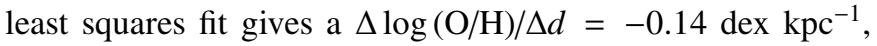
assuming a distance of $840 \mathrm{kpc}$, to be compared with the overall gradient by Vílchez et al. (1988), $\Delta \log (\mathrm{O} / \mathrm{H}) / \Delta d=$ $-0.10 \mathrm{dex} \mathrm{kpc}^{-1}$, assuming the same distance. The Pearson correlation coefficient for our relationship is 0.6. On the other hand our result depends quite strongly on a single PN (8). Therefore the study of more PNe at large galactocentric distances is needed to clarify this.

\subsection{Relationship among chemical abundances}

In Fig. 5a we show the relation between $\mathrm{He} / \mathrm{H}$ and N/O. Chemical abundances are plotted with their errors. The area included inside the dashed lines is the locus of Galactic PNe, derived from Fig. 3 in P 04. Following the recent results by Exter et al. (2004) for both bulge and disc Galactic PNe on N/O vs. $\mathrm{He} / \mathrm{H}$, PNe with low $\mathrm{N} / \mathrm{O}$ also show low $\mathrm{He} / \mathrm{H}$, but at $\mathrm{N} / \mathrm{O}>0.25$ the whole range of $\mathrm{He} / \mathrm{H}$ is sampled. The existence of a relationship between $\mathrm{He} / \mathrm{H}$ and $\mathrm{N} / \mathrm{O}$ has been discussed 

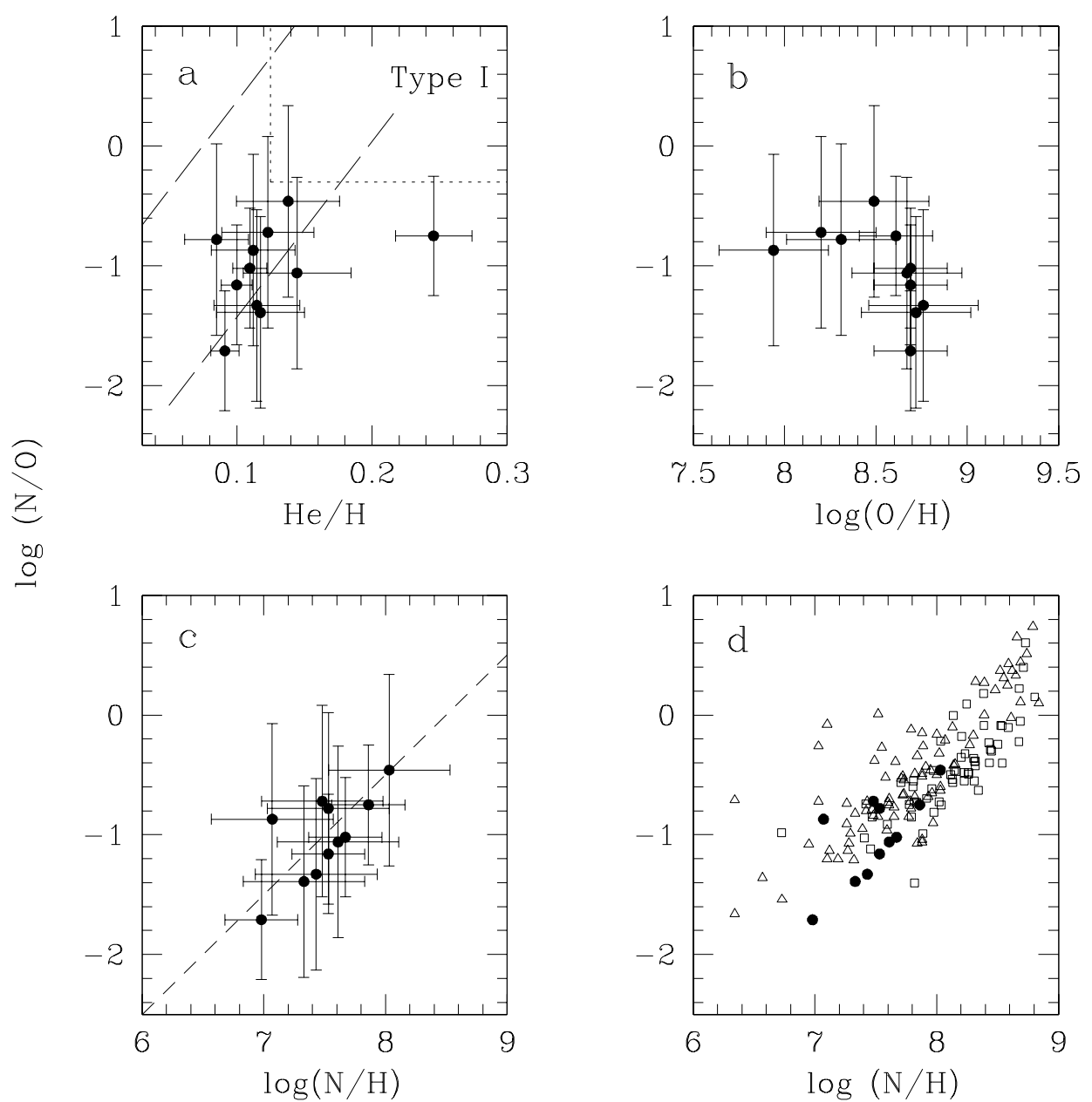

Fig. 5. Some relationships between chemical abundances. a) He/H vs. N/O. The area included inside the dashed lines is the main locus of Galactic PNe derived by Perinotto et al. (2004), hereafter P 04, Fig. 3. The dotted lines show the limits of the type I PNe area. b) O/H vs. N/O: an anti-correlation in shown as seen by JC 99 in M 31; M 33 PNe (present paper) appear to reach lower N/O values than M 31 PNe. c) N/H vs. N/O. The dashed line shows the Galactic relation derived by Henry (1990) as shown by JC 99. In spite of some dispersion, the Galactic relation between N/H and N/O appears to apply also to M 33. d) N/H vs. N/O. Filled circles are PNe of M 33 (this work), triangles are PNe of LMC (Leisy et al. 2004) and squares are Galactic PNe (P 04).

thoroughly by KB 94, who found no correlation, although there might be a correlation in the type I PNe alone. This issue is difficult to assess because there are intrinsic problems in determining the helium abundance such as the collisional corrections and the uncertain ionization fractions. The data of M 33 for $\mathrm{N} / \mathrm{O}$ vs. $\mathrm{He} / \mathrm{H}$ suggest a behaviour similar to the galactic one, but further studies are in order to ascertain this.

Figure $5 b$ shows $\mathrm{O} / \mathrm{H}$ vs. N/O. The existence of an anticorrelation between these quantities is particularly controversial in the literature. Our data indicate a weak anti-correlation, with a Pearson correlation coefficient of 0.5 (0.2 in case of a weighted anti-correlation). Moreover, it appears that, for $12+\log (\mathrm{O} / \mathrm{H})<$ 8.5 , only high values of $\mathrm{N} / \mathrm{O}$ are found, while there is a larger spread for higher $\mathrm{O} / \mathrm{H}$ abundances. The existence of this effect has also been found in Galactic bipolar PNe (Perinotto \& Corradi 1998), in the Galactic bulge (Stasinska et al. 1998), in the SMC (Costa et al. 2000; Leisy \& Dennefeld 2004), possibly in M 31 (JC 99) and the LMC (Leisy \& Dennefeld 2004), although it is not apparent either in the sample of Galactic PNe of KB 94 (covering the range $8.2<12+\log (\mathrm{O} / \mathrm{H})<9.2$ ) or in bulge and disc Galactic PNe of Exter et al. (2004) $(8<12+\log (\mathrm{O} / \mathrm{H})<9.2)$.

In the cases where this transition to high $\mathrm{N} / \mathrm{O}$ ratios for low oxygen abundances is well visible, it occurs at different $\mathrm{O} / \mathrm{H}$ for different galaxies, and roughly at the average overall metallicity computed from other classes of objects like HII regions. This suggests that the explanation offered by Costa et al. (2000), i.e. the dredge-up increasing N/O in the AGB envelope is more efficient at low metallicities, might not suffice, as the transition to high N/O ratios would then always occur at the same metallicity. Together with the fact that in the Galaxy the effect is better visible for high mass progenitors like bipolar PNe (Perinotto \& Corradi 1998), the data would instead point to a high efficiency of the ON cycle in the most massive PN progenitors (Henry 1990), which would lower the $\mathrm{O} / \mathrm{H}$ abundances while increasing $\mathrm{N} / \mathrm{O}$.

In Figs. $5 \mathrm{c}$ and $5 \mathrm{~d}$ the relationships between $\mathrm{N} / \mathrm{O}$ and $\mathrm{N} / \mathrm{H}$ are presented. In Fig. 5c the dashed line represents the correlation found by Henry (1990) for the Galactic PNe. Our data, shown in Fig. 5c with their errors, show some scattering, but the 
Galactic relation between $\mathrm{N} / \mathrm{H}$ and N/O appears to apply to the M $33 \mathrm{PNe}$ as well. The Pearson correlation coefficient is 0.7 . JC 99 suggested an explanation for this correlation: its slope is close to unity and thus the variations of N/O are mainly due to the variation of $\mathrm{N} / \mathrm{H}$ (due to nucleosynthesis of $N$ from progenitors of different mass), while oxygen abundance changes less during the life of PN central stars. In Fig. 5d, N/O vs. N/H is reported for Galactic PNe (P 04), for PNe of the LMC (Leisy \& Dennefeld 2004) and of M 33 (this paper). The PNe of these three galaxies show a common trend.

Now we come to the type I PNe in our sample. The type I PNe, according to the definition of Peimbert \& TorresPeimbert $(1983)$ are those with $\log (\mathrm{N} / \mathrm{O}) \geq-0.3$ and high $\mathrm{He} / \mathrm{H} \geq 0.125$. A more stringent definition was proposed by $\mathrm{KB} 94, \log (\mathrm{N} / \mathrm{O})>-0.1$. However. it has to be noted that the definition of type I PNe should be a function of metallicity, because the amount of $\mathrm{N}$ that can be produced by hot bottom burning is dependent on the amount of $\mathrm{C}$ present and also because the $\mathrm{O} / \mathrm{H}$ abundance depends on the metallicity of the galaxy. Thus a different criterion should be adopted for type I PNe in a galaxy of different metallicity from our Galaxy. The N/O limit above which a PN is a type I, meaning that it has experienced hot bottom burning conversion of $\mathrm{C}$ to $\mathrm{N}$, should be the $\mathrm{C}+\mathrm{N} / \mathrm{O}$ ratio from the $\mathrm{H}$ II regions. This is difficult to estimate since values for $\mathrm{C}$ are hard to obtain. Thus, since the metallicity of M 33 is not so different from that of our Galaxy, we still apply the Galactic criterion to discriminate type I PNe. From Fig. 5a, we note a lack of type I PNe in M33, using the Peimbert \& Torres-Peimbert (1983) criterion. Moreover, we find no type I PNe with the definition by KB 94.

The average $\log (\mathrm{N} / \mathrm{O})$ of $\mathrm{M} 33 \mathrm{PNe}$ is -0.89 , see Fig. 5a. This value is lower than the Galactic non-type I PNe (KB 94) value $(-0.55)$, and it is close to the non-type I PNe value (-0.90; Leisy \& Dennefeld 2004) of the LMC, which is a galaxy of similar metallicity to M 33 (see van den Bergh 2000), whereas Galactic and LMC type I PNe have a higher value $(+0.07$ and +0.02 , respectively, see Table 1$)$.

The number of type I PNe might be connected to the metallicity of the host galaxy. In a deep survey for PNe of the SMC by Jacoby \& De Marco (2002) they found an extremely large number of PNe where the $[\mathrm{N} \mathrm{II}]$ lines are stronger than $\mathrm{H} \alpha$. This might translate into a large type I PN fraction. Comparing the fraction of type I PNe with that in the Galaxy and in the LMC, they suggested that there might be a trend of more type I PNe in lower metallicity galaxies. They explained this trend by the fact that more massive PN central stars, which are progenitors of type I PNe, produce more dust and thus end up being systematically dimmer and consequently harder to detect. This would be more so in a high metallicity galaxy since more dust should be produced. M 33, which has a metallicity intermediate between that of the Galaxy and that of the LMC, might be expected to have a similar number of type I PNe (between $\sim 17 \%$ and $\sim 20 \%$ cf. Jacoby \& De Marco 2002).

An explanation for the smaller number of type I PNe detected might be that type I PNe, which are principally represented by bipolar morphological type and generally associated with younger and more massive progenitors (cf. Corradi \& Schwarz 1995), are less luminous in the [O III] $5007 \AA$ line than

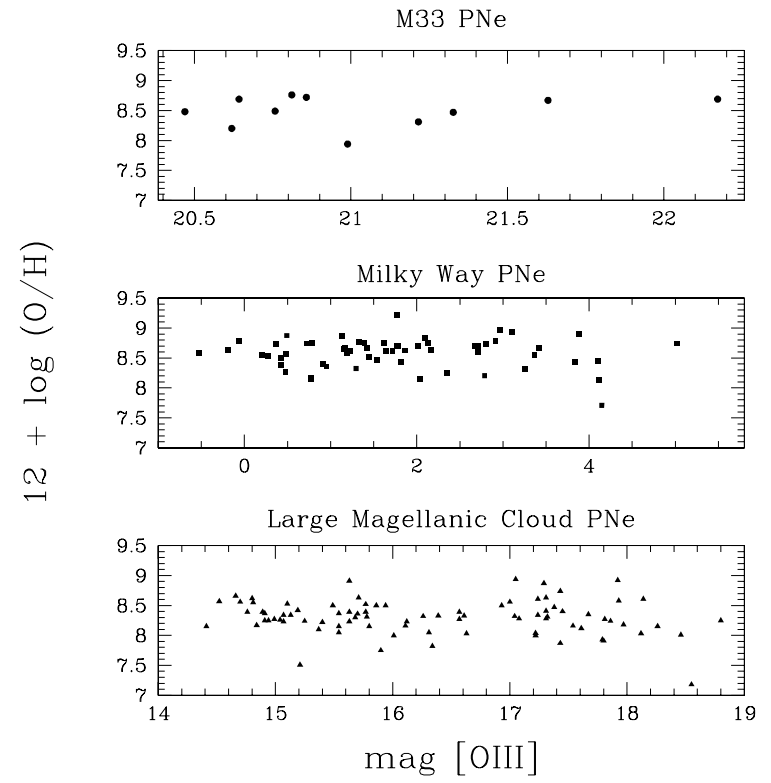

Fig. 6. [O III] luminosity vs. O/H for M $33 \mathrm{PNe}$ (upper panel), MW PNe (central panel) and LMC PNe (lower panel).

other morphological types of PNe (e.g. Magrini et al. 2004, see Figs. 1 and 2, from which one notes that the cutoff of bipolar PNLF is fainter by $\sim 1.5$ mag for the LMC and $\sim 2$ mag for the Galaxy than that of elliptical PNe). We remind the reader that the PNe presented in this paper were discovered in a survey complete up to $\sim 2$ mag fainter than the bright edge of the luminosity function (Magrini et al. 2000). If we consider that the results of luminosity functions of PNe with different morphology presented by Magrini et al. (2004), especially in the case of the Galaxy which has a similar morphology (M 33 is Sc III and the Galaxy is S(B)bc I-II, cf. van den Bergh 2000), apply to M 33 as well, this means that a number of bipolar type I PNe could have been lost because of the incompleteness of the survey; i.e. one should expose more in [O III] to detect type I PNe.

\subsection{Oxygen abundance and [O III] luminosity}

The determination of chemical abundances in an external galaxy rests on its brightest objects. We have examined the $\mathrm{O} / \mathrm{H}$ vs. [O III] luminosity in the M 33 sample, in the LMC, and in the Galaxy to test for possible biases in measuring $\mathrm{O} / \mathrm{H}$ from the brightest PNe (see Fig. 6). The sample of Milky Way PNe also comprises PNe whose chemical abundances have been redetermined by $\mathrm{P} 04$ and $\mathrm{PNe}$ whose distances have been recently obtained by Phillips (2002), whereas the LMC sample consists of PNe with chemical abundances and relative fluxes from the work of Leisy \& Dennefeld (2004), and absolute fluxes from Jacoby et al. (1990).

One might expect that there would be a correlation between the oxygen abundance and the [O III] $5007 \AA$ Auminosity. However, no trend of $\mathrm{O} / \mathrm{H}$ vs. [O III] luminosity is seen in the three galaxies, as already noted for M 31 by JC 99 . There might be various reasons for this. The basic point is that oxygen is one of the most important coolants of the nebula so that a high $\mathrm{O}$ concentration lowers the nebular temperature and consequently 
the flux coming from the $[\mathrm{O}$ III] $5007 \AA$ line. There is therefore a feedback effect from a higher $\mathrm{O} / \mathrm{H}$ concentration and a consequently lower $T_{\mathrm{e}}$ which produces the mentioned lack of trend of $\mathrm{O} / \mathrm{H}$ vs. [O III] luminosity. This means that $\mathrm{O} / \mathrm{H}$ derived from the brightest $\mathrm{PNe}$ (as from any $\mathrm{PN}$ ) is representative of the total PNe population. This is particularly useful when deriving chemical abundances for PNe belonging to external galaxies, where only the brighter tip of their luminosity function is observed.

\section{Summary and conclusions}

Using the CLOUDY photoionization code, we have derived nebular parameters and some stellar effective temperatures for $11 \mathrm{PNe}$ belonging to the spiral galaxy M 33, and measured their $\mathrm{He} / \mathrm{H}, \mathrm{O} / \mathrm{H}, \mathrm{N} / \mathrm{H}, \mathrm{Ar} / \mathrm{H}$ and $\mathrm{S} / \mathrm{H}$ abundances. The data are consistent with the $\mathrm{O} / \mathrm{H}$ gradient from $\mathrm{H}$ II regions by Vílchez et al. (1988), but abundances in PNe farther from the centre of M 33 than the sample studied here are needed to confirm a metallicity gradient from $\mathrm{PNe}$ in $\mathrm{M}$ 33. A trend of N/O to N/H similar to that for the Galactic PNe. We did not find PNe with the very high $\mathrm{N} / \mathrm{H}$ and N/O values that are typical of galactic type I PNe. A possible explanation is that the incompleteness of the survey for M 33 PNe (M 00) caused the loss of type I PNe (N/O > -0.3), which are generally faint in the [O III] $5007 \AA$ emission line. Finally we found that the [O III] luminosity is clearly independent of the oxygen abundance. Therefore it is possible to use every PN, and particularly the brightest $\mathrm{PNe}$, in external galaxies as representative for the whole PN population.

Acknowledgements. We especially thank Dr. Roberto Baglioni for his precious help in writing the Perl procedure. We are extremely grateful to an anonymous referee for comments and useful suggestions that improved the paper significantly.

\section{References}

Armsdorfer, B., Kimeswenger, S., \& Rauch, T. 2002, RMxAC, 12, $180 \mathrm{I}$

Ambartsumian, V. A. 1932, Poulkovo Obs. Circ, 4, 8

Che, A., \& Köppen, J. 1983, A\&A, 118, 107

Corradi, R. L. M., \& Schwarz, H. 1995, A\&A, 293, 871

Costa, R. D. D., de Freitas Pachego, J. A., \& Idiart, T. P. 2000, A\&AS, 145,467
Dennefeld, M. 1989, in Recent Developments of Magellanic Cloud Research, ed. K. S. de Boer, F. Spite, \& G. Stasinska (Meudon: Publ. Observatoire de Paris), 107

Dufour, R. J. 1984, in Structure and evolution of the Magellanic Clouds, ed. D. Reidel (Dordrecht: Reidel), IAU Symp., 108, 353

Exter, K. M., Barlow, M. J., \& Walton, N. A. 2004, MNRAS, 349, 1291

Ferland, G. J., Korista, K. T., Verner, D. A., et al. 1998, PASP, 110, 761

Gurzadyan, G. A. 1988, Ap\&SS, 149, 343

Gurzadyan, G. A. 1997, The Physics and Dynamics of Planetary Nebulae (Berlin: Springer), 112

Henry, R. B. C. 1990, ApJ, 356, 229

Howard, J. W., Henry, R. B. C., \& McCartney, S. 1997, MNRAS, 284, 465

Iben, I. Jr., \& Renzini, A. 1983, ARA\&A, 21, 271

Jacoby, G. H., \& Ciardullo, R. 1999, ApJ, 515, 169 (JC 99)

Jacoby, G. H., \& De Marco, O. 2002, AJ, 123, 269

Jacoby, G. H., Walker, A. R., \& Ciardullo, R. 1990, ApJ, 365, 471

Kingsburgh, R. L., \& Barlow, M. J. 1994, MNRAS, 271, 257

Leisy, P., \& Dennefeld, M. 2004, in preparation

Leisy, P., Dennefeld, M., \& Francois, P. 2000, in Proceeding of Ionized Gaseous Nebulae, Mexico City November 21-24, 2000, 32

Magrini, L., Corradi, R. L. M., \& Mampaso, A., \& Perinotto, M. 2000, A\&A, 355, 713 (M 00)

Magrini, L., Perinotto, M., Corradi, R. L. M., \& Mampaso, A. 2003, A\&A, 400, 511 (M 03)

Magrini, L., Corradi, R. L. M., Leisy, P., et al. 2004, in Asymmetric Planetary Nebulae III, ASP Conf. Ser., in press

Maran, S. P., Gull, T. R., Stecher, T. P., Aller, L. H., \& Keyes, C. D. 1984, ApJ, 280, 615

Morisset, C. 2004, AJ, 601, 858

Peimbert, M., \& Torres-Peimbert, S. 1983, in Planetary Nebulae, ed. D. R. Flower (Dordrecht: Reindel), IAU Symp., 103, 233

Perinotto, M., \& Corradi, R. L. M. 1998, A\&A, 332, 721

Perinotto, M., Morbidelli, L., \& Scatarzi, A. 2004, MNRAS, in press (P 04)

Phillips, J. P. 2002, ApJS, 139, 199

Rauch, T. 2003, A\&A, 403, 709

Richer, M. G., \& McCall, M. 1995, ApJ, 445, 642

Stasinska, G., Richer, M. G., \& Mc Call, M. L. 1998, A\&A, 336, 667

van den Bergh, S. 2000 in The Galaxies of the Local Group (Cambridge University Press)

Vílchez, J. M., Pagel, B. E. J., Diaz, A. I., Terlevich, E., \& Edmunds, M. G. 1988, MNRAS, 235, 633

Walsh, J. R., Dudziak, G., Minniti, D., \& Zijlstra, A. A. 1997, ApJ, 487,651

Walsh, J. R., Walton, N. A., Jacoby, G. H., \& Peletier, R. F. 1999, A\&A, 346, 753 